\title{
Semi-automatic Sea Lane Extraction Combining Particle Filtering (PF) and Geographic Information Systems (GIS)
}

\author{
Katerina TZAVELLA ${ }^{1}$ and Martin ULMKE ${ }^{2}$ \\ ${ }^{1}$ Fraunhofer FKIE, Wachtberg / Germany · katerina.tzavella@fkie.fraunhofer.de \\ ${ }^{2}$ Fraunhofer FKIE, Wachtberg / Germany
}

This contribution was double-blind reviewed as full paper.

\begin{abstract}
This paper addresses the problem of extracting traffic patterns from noisy and incomplete sensor data. We introduce an approach that combines the output of (particle filter based) tracking filters with GIS techniques. While tracking filters are standard tools for generating estimates of the position and velocities of individual objects over time, GIS techniques are used to extract geographic patterns from these estimates. GIS techniques also allow the integration of prior knowledge, such as coastlines and bathymetry, to enhance the quality of the extracted patterns. The approach has been applied to maritime traffic surveillance using report of the Automatic Identification System (AIS), which may be subject to GPS errors, spoofing, time delays and periods of missed detections. The results are continuous vessel tracks indicating each vessel's route, even when the update rate of messages is significantly slower.
\end{abstract}

\section{Introduction}

There is an increasing need for an enhanced situation awareness of maritime traffic both for open sea and coastal areas. The requirement for maritime surveillance and monitoring is to foster safety and security of maritime traffic, e.g., to avoid collisions and other hazards, and to quickly detect accidents, oil spills, and illegal activities. Multi-sensor fusion and tracking can significantly enrich the consolidated surveillance picture, and provide an effective basis for anomaly detection processing (CARTHEL et al. 2007).

The extraction of traffic patterns from sensor data has attracted increasing attention with applications in different environments. In particular, for maritime surveillance, traffic patterns can be used for vessel route prediction and anomaly detection. Even when collaborative report data, Automatic Identification System (AIS), is available, traffic pattern extraction can be impeded by sensor errors, spoofing, and periods of missed detection due to limited coverage, terrain masking, or technical issues. The extraction and update of tracks of individual objects using noisy and erroneous sensor data is the task of tracking filters (BAR-SHALOM et al. 2004, KосH 2010). From the time series of a large set of 
individual tracks, traffic patterns can be extracted (RISTIC et al. 2008, VeSPE et al. 2012, Pallota et al. 2013, Perez et al 2009, AARSÆTHER 2011).

This paper describes an extended version of the road map extracting approach discussed in TZAVELla \& UlmKe (2013), which here is applied to the problem of maritime pattern extraction.

\section{Input Data and Data Processing}

The area under investigation is a marine area of $6156 \mathrm{~km}^{2}(54 \mathrm{~km} \times 114 \mathrm{~km})$ of the Adriatic sea, outside of Venice in Italy and the AIS data, provided by the EU-funded project NEREIDS (https://www.nereids-fp7.eu) are spread in this area (see figure 1). The AIS data were at first visualized in an ArcMap environment as georeferenced points (longitude and latitude). They are also carrying information on the speed of each vessel, COG (Course Over Ground), SOG (Speed Over Ground), as well as date and time of message generation. However, we only used the position and the dates of message propagations from the AIS data provided and only for three data vessels (see figure 2). The positions of these vessels were ground truth data for further investigation and were selected taking into account their MMSI (Maritime Mobile Service Identity) and the date of signal arrival. An MMSI number is a unique identifier assigned to a boat. Only one number is assigned for all applicable electronics on the vessel, such as an AIS transponder etc.

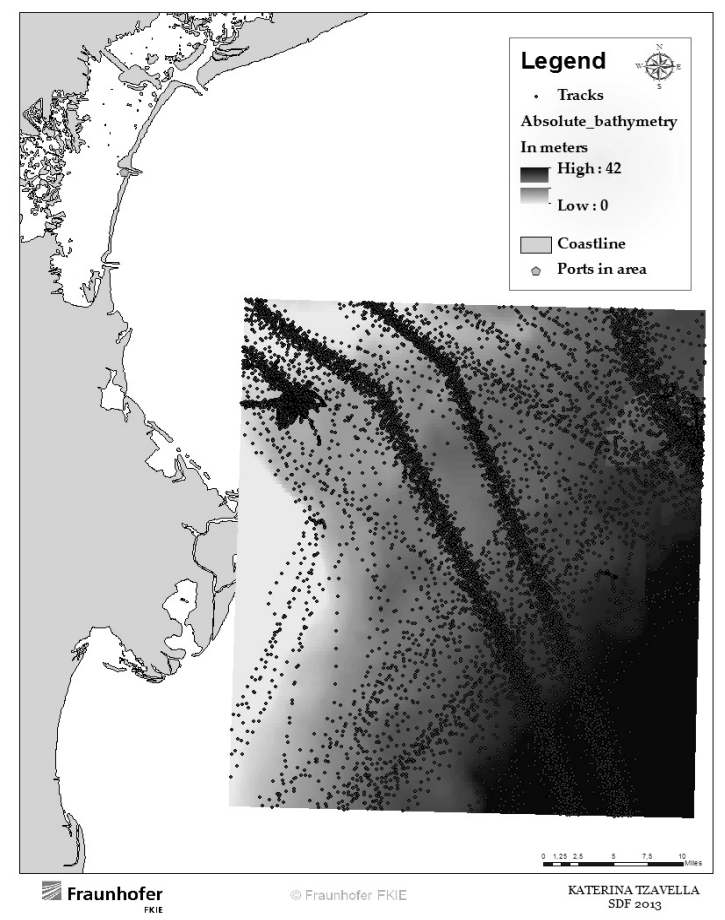

Fig. 1:

Absolute bathymetry of the under study area and the AIS data provided by NURC 


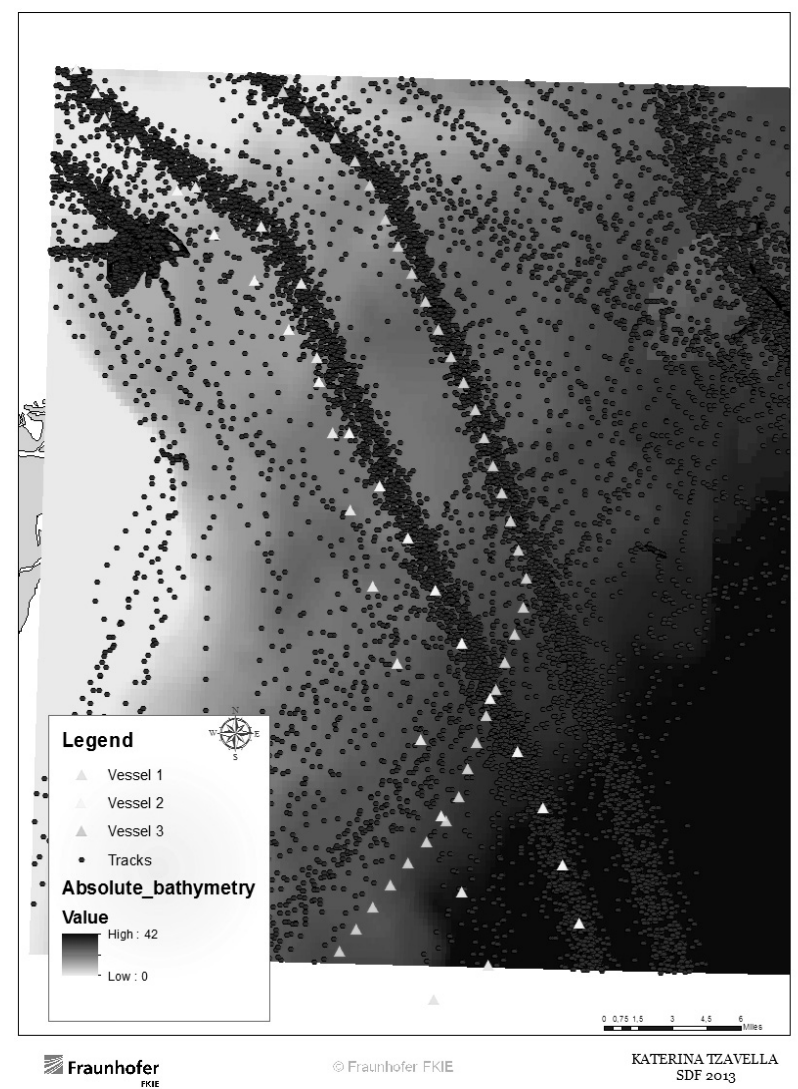

Fig. 2: The three vessels selected as ground truth data

To test if the proposed methodology is working in cases of missed detections, we decreased the update rate of messages received from vessels 2 and 1 (larger gaps in between the messages). The messages seem to propagate significantly slower than those from vessel 3 (see figure 2).

\section{Methods for Sea Lane Extraction}

\subsection{Particle cloud extraction - First part of the algorithm}

In this work we apply a sequential Monte Carlo technique, also known as Particle Filter, and more precisely the "sequential importance resampling (SIR)" algorithm (GORDON et al. 1993). Particle filters allow the treatment of highly non-linear target and/or measurement models. The result of the particle filter is a time series of a particle cloud that serves as an estimate for the target state (position and velocity) at each instant of time where a filter update has been performed, typically after each measurement (message propagation to the AIS). From the particle cloud, the coordinates of the underlying path are estimated as 
described below. The technique is applied to simulated sea lane traffic in different scenarios such as straight lanes, cross section, and turns. The messages are generated using typical parameters for vessels. Then we extract the positions of each particle for every time step into a matrix. Specifically, the results of this part of the algorithm are the particles $(500$, in this example) spread around each position of the ground truth, and furthermore a cloud of particles indicating the area of sea lane / path existence (see figure 3).

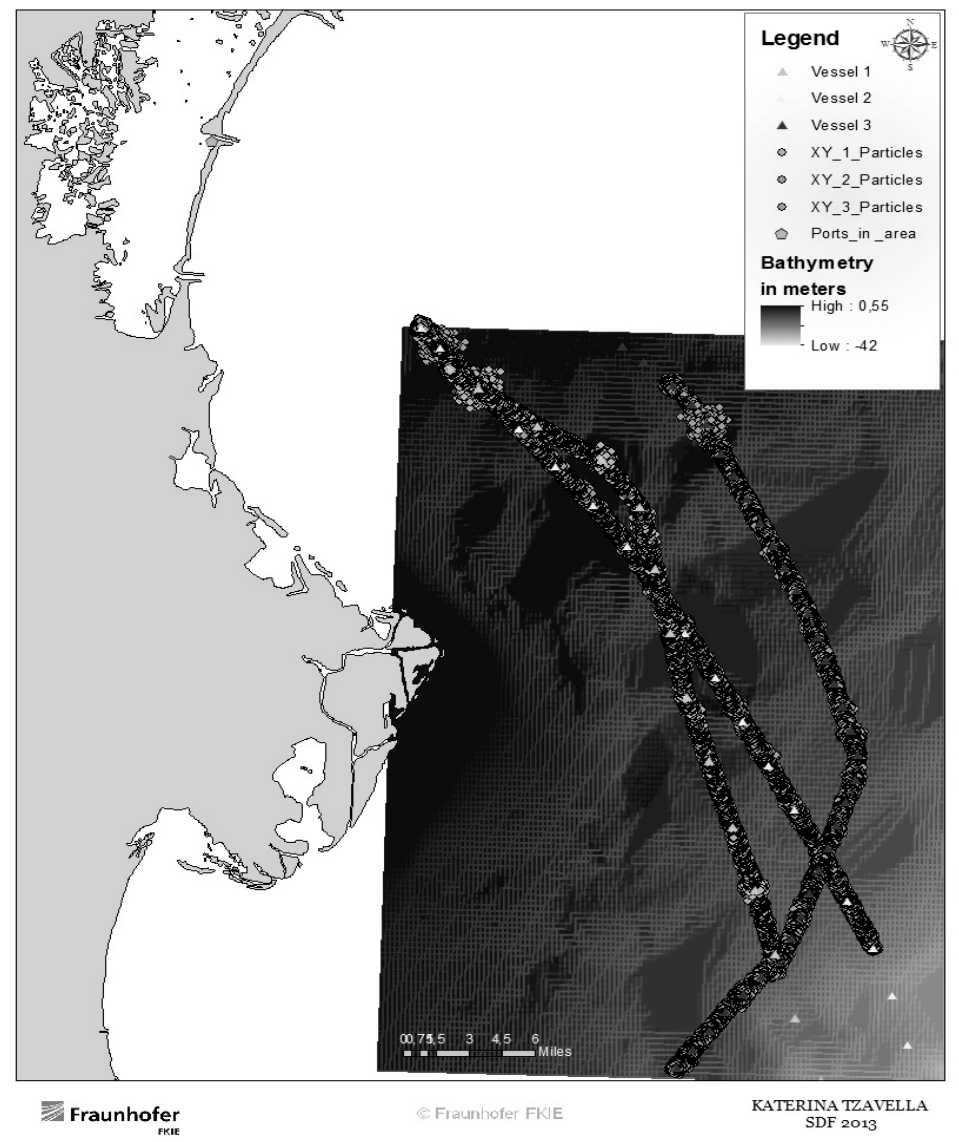

Fig. 3: Particle clouds extracted for each vessel indicative of sea lanes followed by them

\subsection{Sea lane extraction - Second part of the algorithm}

The aforementioned clouds of particles are used as input data for the second part of the algorithm which involves different kinds of GIS methods (see figure 4). The clouds of particles, created by particle filtering of each ground truth in different scenarios, are added to the ArcMap (version 10.1) environment by displaying their positions indicated by longitudes and latitudes (X and $\mathrm{Y}$ ). 


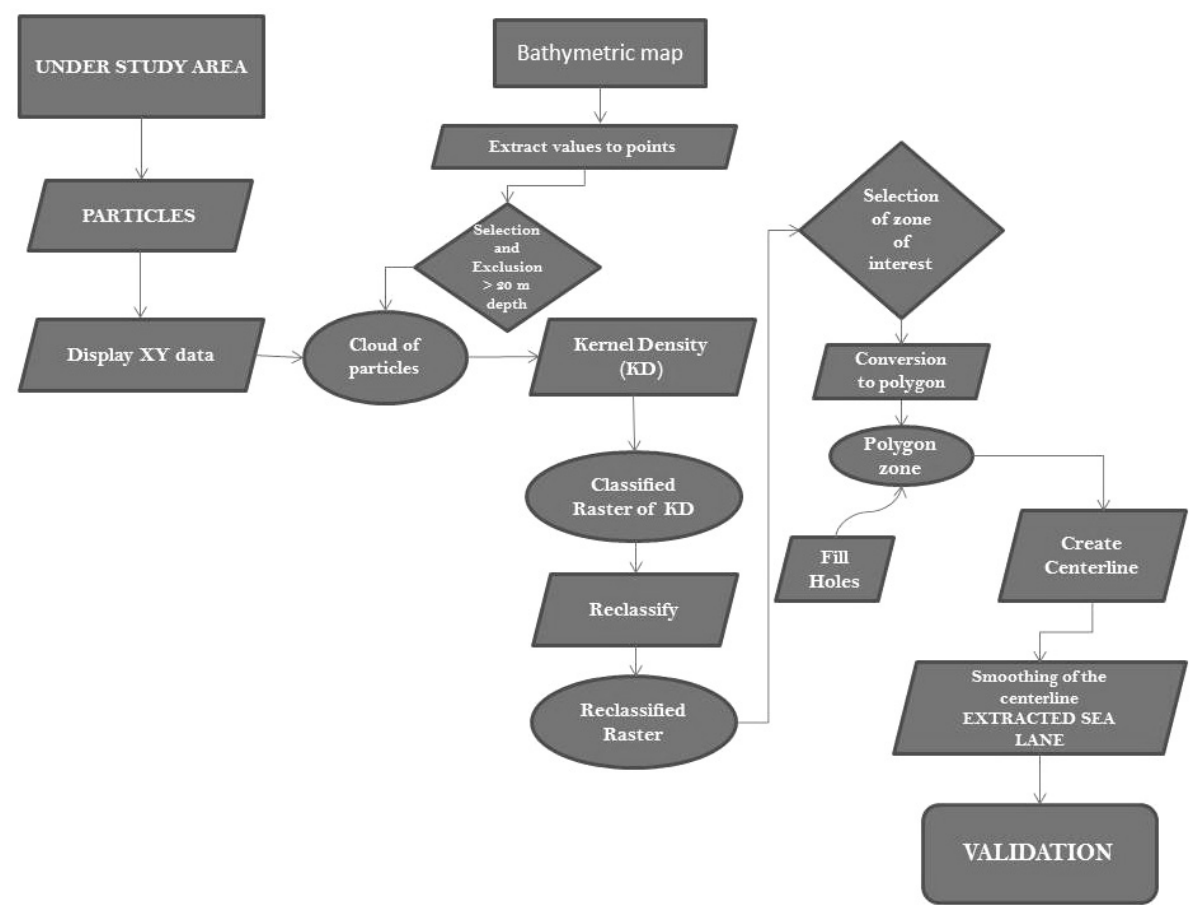

Fig. 4: The methodology followed in a GIS environment for sea lane extraction

In general, kernel methods estimate densities within a neighborhood around each output raster cell (in our case a circular one). Conceptually, a smoothly curved surface is fitted over each particle. The surface value is the highest at the location of the ground truth points and diminishes with increasing distance from them, reaching zero at the Search radius distance of 2 kilometers from the ground truth points. The volume under the surface equals the population field value for the point. The population field could be used to weight some features higher than others, depending on their meaning, or to allow one point to represent several observations. The density at each output raster cell is calculated by adding the values of all kernel surfaces where they overlay the raster cell center, and can be presented also by percentage of the total amount. The kernel function is based on the quadratic kernel function described in GIBIN et al. (2007, 76, eq. 4.5).

This resulting raster file is indicative of areas with high density of particles and for this reason the use of a threshold is essential to make the methodology more robust and suitable for different scenarios. The threshold for this algorithm was identified via the "Standard Deviation Classification" excluding the value of zero, resulting in a raster indicative of the density of the particles in each under study area, classified in 5 zones/classes (see figure 5).

The threshold is obvious in figure 6, where the first class break of the classes/zones of densities of particles is the "critical value" since it divides the density into two critical zones: One with $90 \%$ of particles on the right side of the critical value and the other with $10 \%$ on the left side of it. For further analysis we keep these two zones and we create a binary raster representing the areas of higher and lower density whereas the latter will not 
be useful for further analysis. This binary raster resulted after reclassifying the raster of KDE with the tool "Reclass" in the Spatial Analyst toolbox of ArcMap 10.1. The density zone of the higher percentage of particles was converted into a polygon shapefile. Any holes (areas of NODATA - no provided information) observed in the polygon were filled through a set of functions in "ET Geo Wizard", which ensures topological correctness of a polygon feature data set.

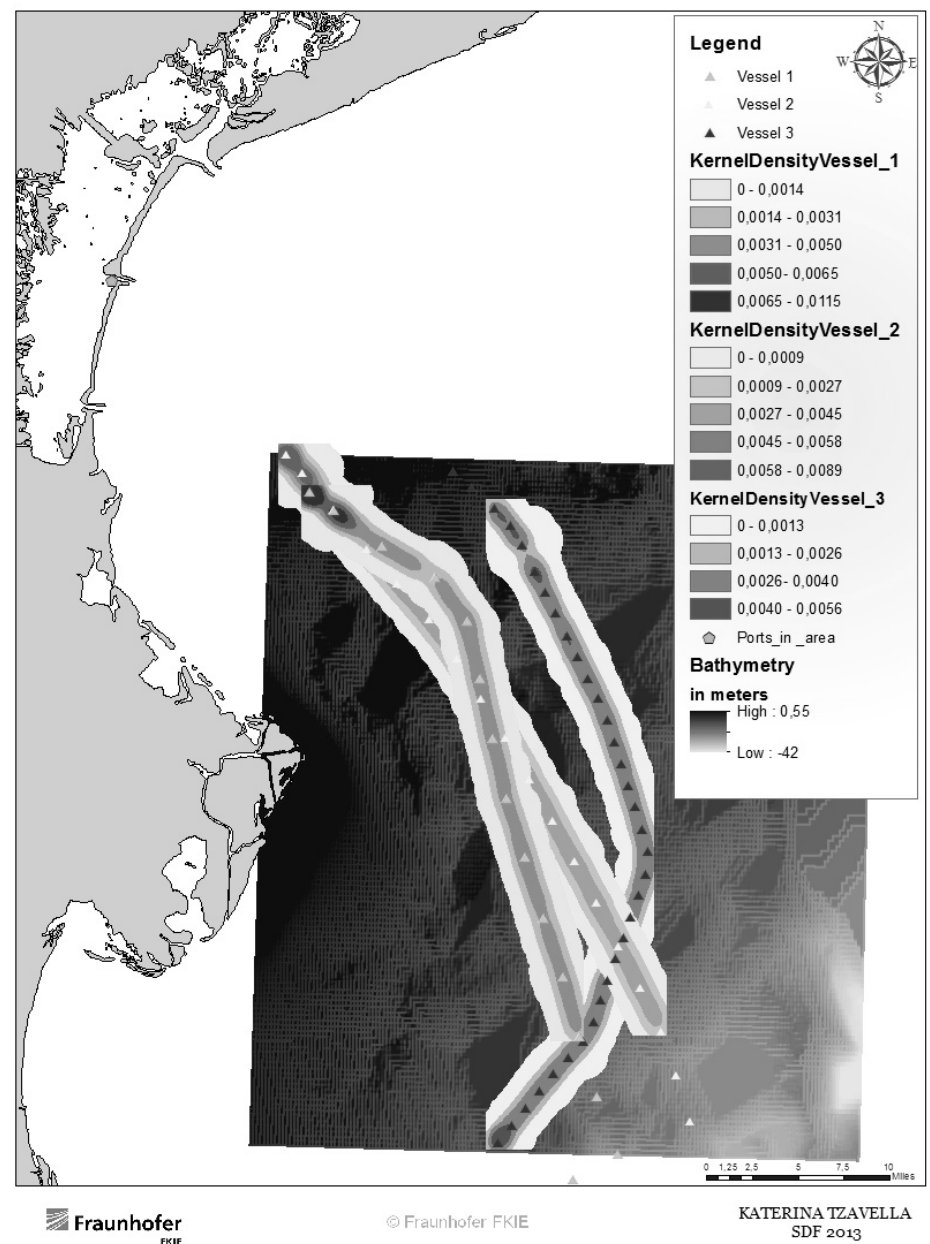

Fig. 5: Kernel density rasters of $100 \mathrm{~m}$. Spatial analysis for each vessel visualized with the bathymetric data of this area. 


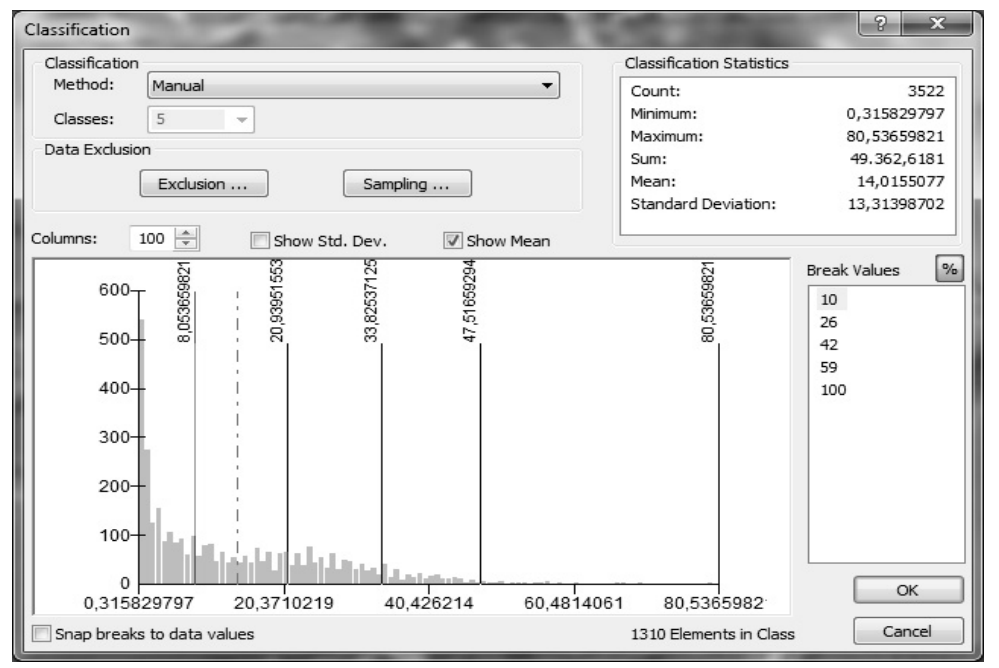

Fig. 6: Histogram of standard deviations from the mean value of particles (dashed line). The red line is the "critical value".

In addition, we spatially selected and excluded particles existing in an area of less than 1520 meters of depth, where vessels in this study area cannot exist. This exclusion of particles reduces the computation time and limits the probability density to regions that are feasible. In a last step the "ET Geo Wizard" and specifically the function "Create Centerline" was used to create a center line inside the polygon. This line is passing through points which are equally distant from each side of the polygon and is the path that we are aiming at. The only restriction of the algorithm of center line extraction is the selection of the minimum and maximum width of the polygon (higher probability detection zone). We used as minimum width the value of 50 meters and maximum width the value of 1500 meters, which are approximately the min. and max. width of sea lanes concerning the width of existing vessels. For a better fitting result with a smooth extracted sea lane that is close to the ground truth points, we used the smoothing method of "Bezier curve" provided by the function "Smooth". This line can now be considered as our extracted sea lane, which is close to the chosen vessel trajectories' positions (close to the ground truth points depicted in figure 8).

\section{Results}

Validation ensures that the model meets its intended requirements in terms of the methods employed and the results obtained. The ultimate goal of model validation is to make the model useful in the sense that the model addresses the right problem, provides accurate information about the system being modelled, and to make the model actually used. The results of this methodology are the sea lanes followed by the three selected vessels. The validation will occur after visualization of the data (extracted sea lanes and ground truth positions of the selected vessels). As shown in figure 7, these extracted sea lanes are very 
close to the ground truth data, which means that the proposed methodology is successfully implemented giving the desirable results.

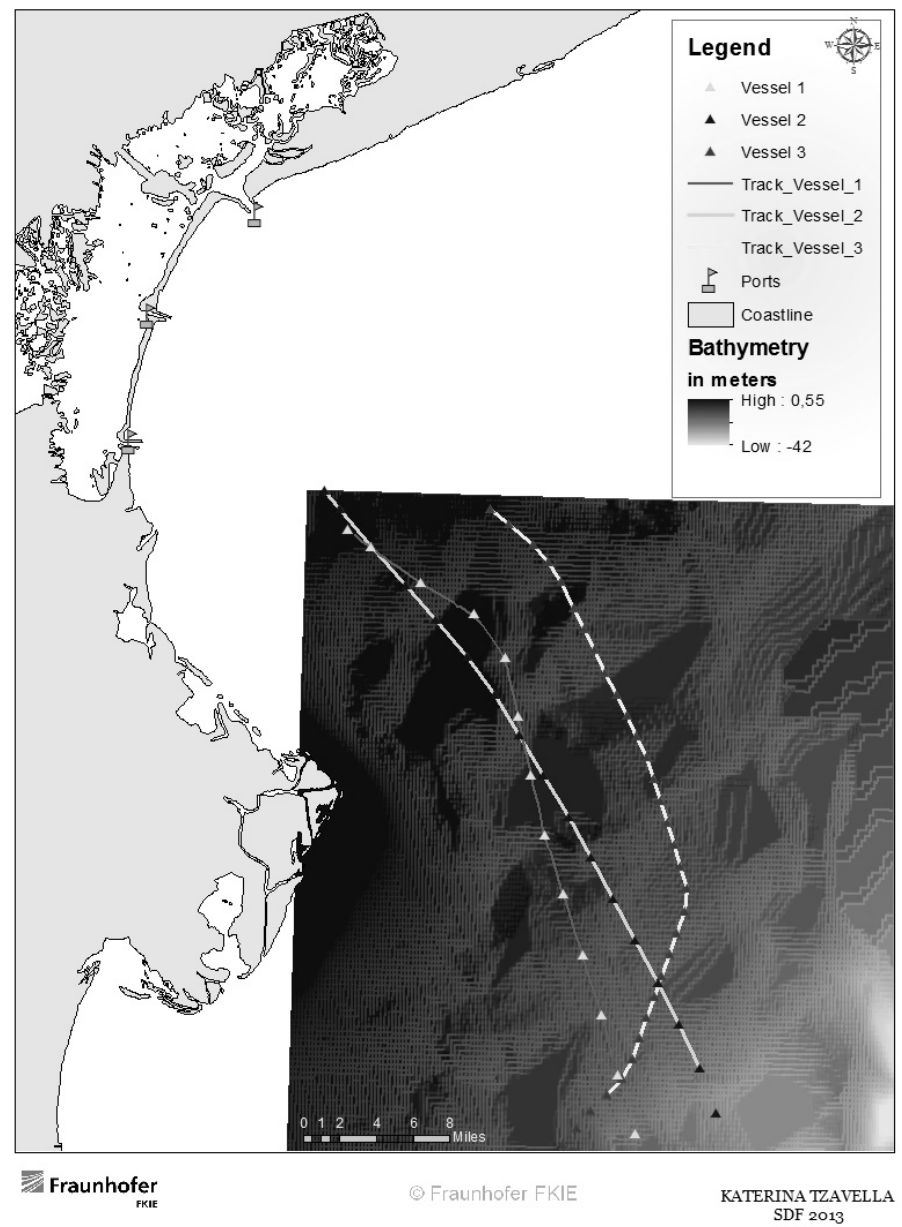

Fig. 7: Extracted sea lanes matching the trajectories of vessels which were selected as our ground truth data.

A closer look at the cases with missed detections / delayed received messages (vessels 1, 2) reveals in figure 8 that the methodology suggested in this paper is working well since the result is a continuous sea lane. Even after decreasing the update rate of signal propagation (one message too far from the next one) the model was able to give a continuous line, which in this case study is representative of a sea lane, without any discontinuities. 


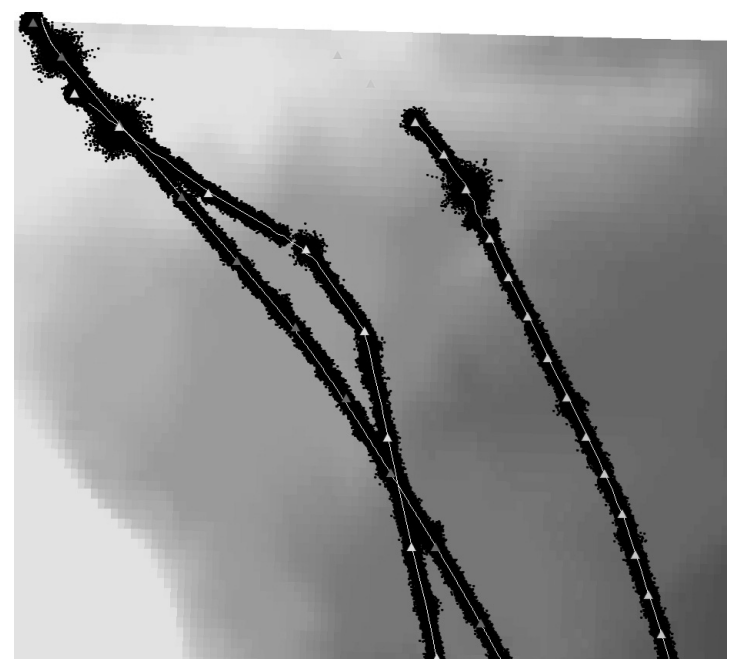

Fig. 8:

Continuous yellow lines / extracted sea lanes in cases of missed detections for vessels 1,2 (distance from one signal propagation to another more than $4 \mathrm{~km}$ on the left) in comparison with typical (every $2 \mathrm{~km}$ ) signal propagation from the vessel 3 on the right.

\section{Conclusion and Outlook}

While the Terrestrial AIS service provides near real-time updates of vessel positions at the areas covered by the coastal AIS receivers' network, the SAIS (Satellite AIS) service provides position updates less often, at intervals that may vary from a few minutes up to several hours. On average, several SAIS updates per day should be expected for most vessels sailing the oceans. In such cases, the combination of particle filtering of selfreported AIS data from targets moving in a marine area and GIS methods seem to be a powerful tool for the extraction of sea lanes as well as in cases of missed detections. The reduction of the amount of particles used by the proposed methodology leads to cost minimization and to a better accuracy of the results (extracted sea lanes close to the ground truth points of the chosen vessels' trajectories). We also achieved a holistic and successful exploitation of tracking results and data information, since the algorithm described in this paper is open to several extensions (e.g. automatization, multiple target tracking etc.). The current limitations are the prior knowledge of the environment of the area under investigation, the bathymetry of the area (bathymetric raster), or the already existing sea lane network within ports, passages, canals etc. Another advantage of this methodology is its high degree of automatization. 


\section{References}

AARSÆTHER, K. G. (2011), Modeling and analysis of ship traffic by observation and numerical simulation. Doctoral dissertation, NTNU.

Bar-Shalom, Y., X. Rong L. \& Kirubarajan, T. (2004), Estimation with applications to tracking and navigation: theory algorithms and software. John Wiley \& Sons.

Gibin, M., Longley, P. \& AtKinson, P. (2007), Kernel Density Estimation and Percent Volume Contours in General Practice Catchment Area Analysis in Urban Areas. Proc. Geographical Information Science Research Conf.

Gordon, N. J., Salmond, D. J. \& Smith, A. F. M. (1993), Novel approach to nonlinear/non-Gaussian Bayesian state estimation. IEEE Proceedings $\mathrm{F}$ on Radar and Signal Processing, 140 (2), 107-113.

KocH, W. (2010), On Bayesian tracking and data fusion: a tutorial introduction with examples. Aerospace and Electronic Systems Magazine, IEEE, 25 (7), 29-52.

Pallotta, G., Vespe, M. \& BRyAn, K. (2013), Vessel Pattern Knowledge Discovery from AIS Data: A Framework for Anomaly Detection and Route Prediction, NATO Undersea Research Centre Viale San Bartolomeo 400, La Spezia, Italy.

Perez, M., Chang, R., Billings, R. \& Kosub, T. L. (2009), Automatic Identification System (AIS) data use in marine vessel emission estimation. 18th Annual International Emission Inventory Conference.

Ristic, B., La Scala, B., Morelande, M. \& Gordon, N. (2008), Statistical analysis of motion patterns in AIS data: anomaly detection and motion prediction. $11^{\text {th }}$ International Conference on Information Fusion, 1-7.

Vespe, M., BRyAn, K. BRACA, P. \& Visentini, I. (2012), Unsupervised learning of maritime traffic patterns for anomaly detection. 9th IET Data Fusion \& Target Tracking Conference (DF\&TT'12), London, UK, 16-17 May. 